\title{
Subaortic Stenosis Same Disease Different Presentation Review of Three Cases
}

\author{
Hernández Pérez JE, Delgado Leal L*, Lugo R, Botti A, Marín D, Salas J and Rodríguez R \\ Centenario Hospital Miguel Hidalgo, México
}

*Corresponding author: Delgado Leal L, Cardiology Departament, Centenario Hospital Miguel Hidalgo, Aguascalientes, Aguascalientes, México

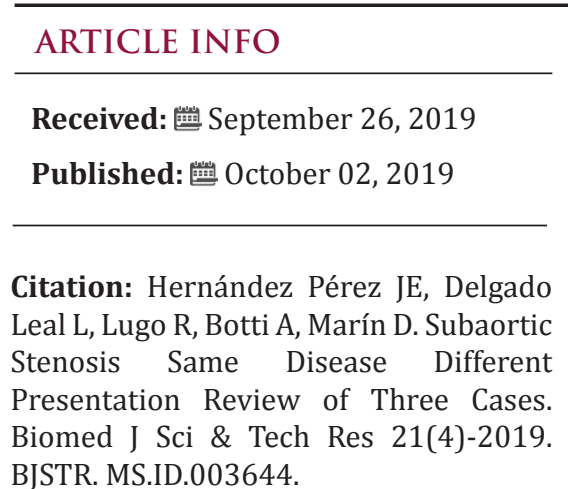

ABSTRACT

Keyword: Cardiopathic; Aorta; Murmur

\section{Introduction}

Sub valvular aortic stenosis (SAS) is the second most common type of aortic stenosis, representing $14 \%$ of the obstruction in the left ventricular outflow tract (LVOT), with valvular aortic stenosis being the most common cause (70\%). The prevalence is $6.5 \%$ of all congenital heart diseases in adults [1], it can occur as a discrete membrane under the aortic valve in the left ventricular outflow tract causing a tunnel-shaped obstruction, as a result of chordal fixation during the development stage [2]. We present three clinical cases in adult patients:

\section{Case 1}

31-year-old man history of stroke in childhood. He starts with progressive dyspnea functional class II 6 months ago. Physical examination shows a meso systolic aortic murmur II / IV of ejection intensity without irradiation, and symmetrical radial pulses. TEE (transesophageal echocardiogram) (Figure 1) Diastolic diameter LV (left ventricle) $47 \mathrm{~mm}$, DSVI (systolic diameter of the left ventricle) 30, VTD (tele diastolic volume) 170, LVEF (left ventricular ejection fraction) 53\%, without segmental mobility alterations. Trivalve aortic valve with subaortic impeller of $5 \mathrm{~mm}$ to $2 \mathrm{~mm}$ of the ring, maximum speed $2.6 \mathrm{~m} / \mathrm{s}$, maximum gradient of 27 , average gradient of $14 \mathrm{~mm}$.

\section{Case 2}

21-year-old woman with a history of persistence of arterial duct without corrective treatment. She begins 7 months ago with a decrease in functional class II-III, accompanied by lower limb edema, presenting clinically short stature, neck with IY grade II/ IV, bilateral carotid murmur II/IV, hepatojugular reflux (+) positive thrill irradiated to the neck. Apical impulse precord displaced to the 6th IBD, low parasternal lift with palpation of the closure of the pulmonary, meso systolic aortic murmur III/ IV intensity in increasing with late acme radiated to the neck. Sisto-diastolic pulmonary epicenter murmur in rough IV/ IV tone of irradiated intensity to the left and axillary infraclavicular region, meso systolic mitral epicenter murmur II/IV of aspirated intensity irradiated to the left axillary region. TTE (transthoracic echocardiogram): (Figure 2) Severe dilatation of the atrium and left ventricle, Grade 1 diastolic dysfunction, moderate systolic dysfunction of the LVEF of $38 \%$, valvular: severe aortic stenosis due to subaortic fibromuscular impeller, moderate pulmonary hypertension with persistent arterial duct the incidence of the association of sub-ortic stenosis and persistence of artery duct as the case presented is $34 \%$ reported in case series [3]. 


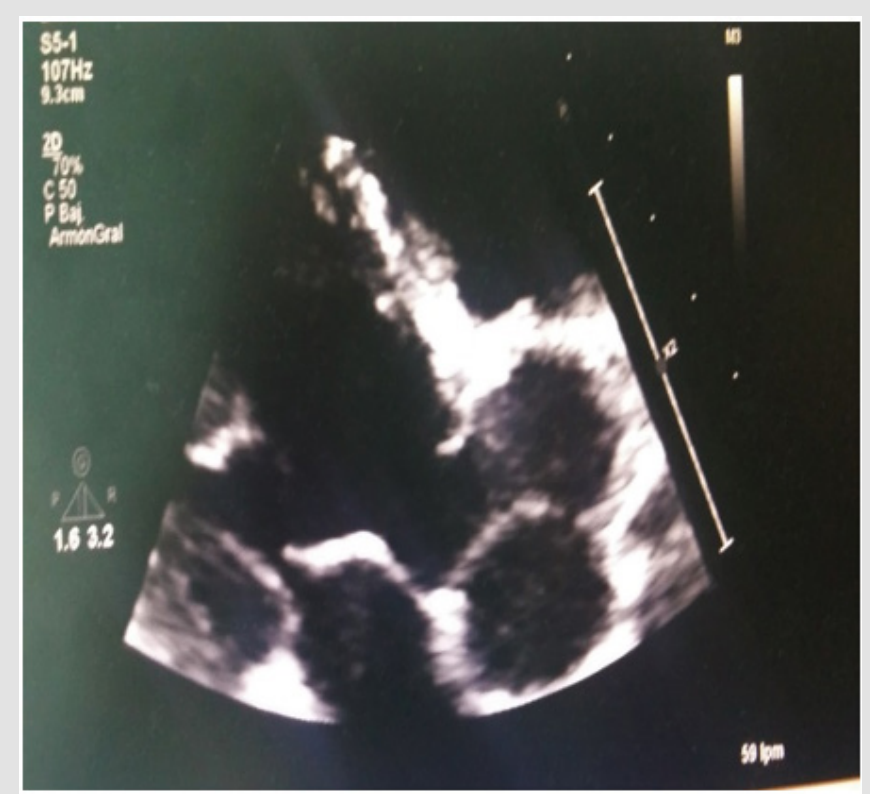

Figure 1: TEE $80^{\mathrm{a}}$ subaortic impeller of $5 \mathrm{~mm}$.

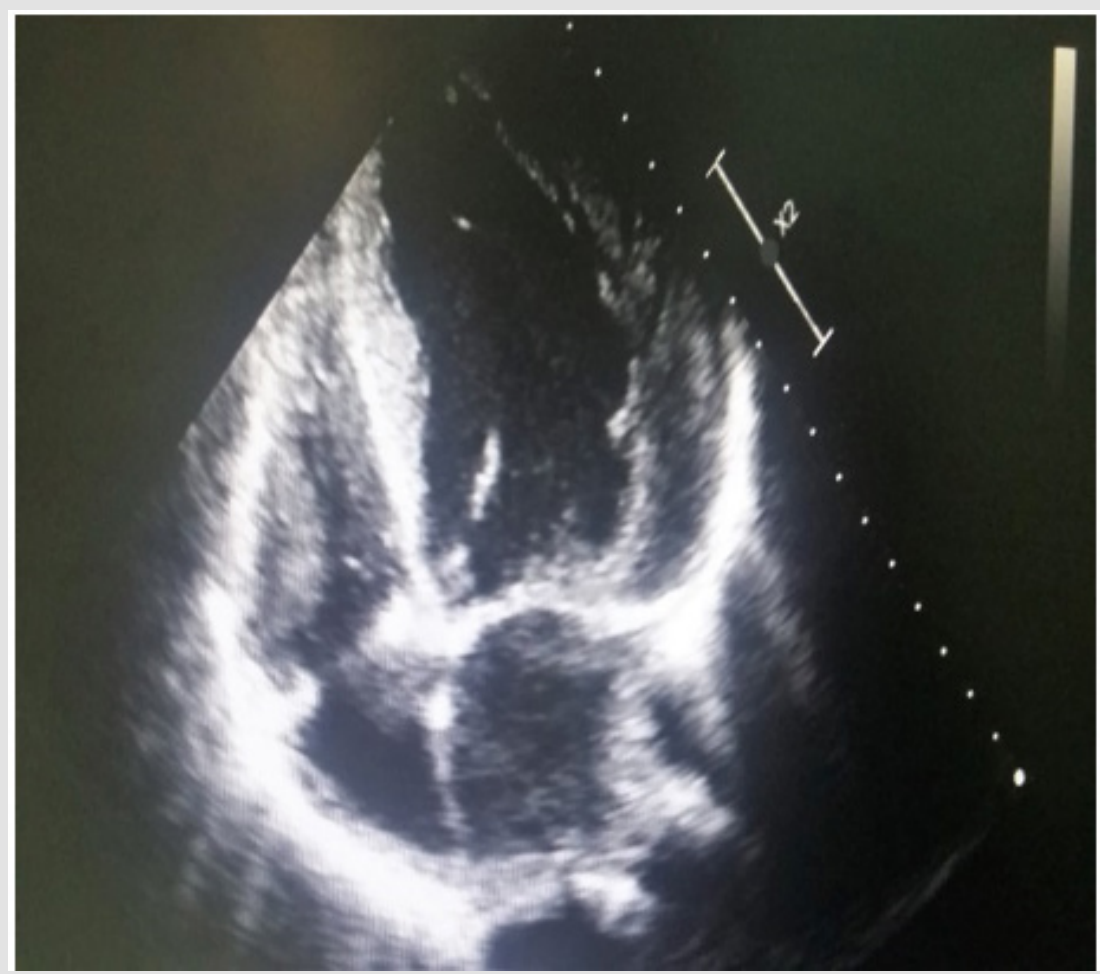

Figure 2: TTE Four -chamber subaortic fibromuscular impeller with persistent arterial duct.

\section{Case 3}

30-year-old woman who has a history of sub-aortic stenosis and persistent arterial duct heart disease. Arterial duct closure, she had surgery at 5 years, she is now being treated with Captopril and spironolactone. It is assessed by a decrease in functional class II / III of 8 months of evolution, moderate throbbing-type headache as well as regular palpitations of sudden onset accompanied by dyspnea,1 month ago she introduced a cardiogenic type syncope, EKG with LVH and systolic overload (Figure 3) TTE (Figure 4) Dilatation of LV with mixed hypertrophy. Systolic dysfunction of
LVEF 30\% generalized hypokinesia. Ascending aorta of $45 \mathrm{~mm}$, double lesion severe stenosis $0.6 \mathrm{~cm}$, speed of $5.17 \mathrm{~m} / \mathrm{s}$, maximum gradient of $107 \mathrm{~mm} \mathrm{hg}$, medium gradient $78 \mathrm{~mm} \mathrm{hg}$, contracted vein of $6 \mathrm{~mm}$, ORE (external regurgitation hole) 0.38, aortic angiography was requested due to aortic dilation ascending (Figure 5) reporting aorta with $21 \mathrm{~mm}$ root, $31 \mathrm{~mm}$ Valsalva sinus, $45 \mathrm{~mm}$ ascending aorta, finding a common brachiocephalic trunk with bovine aortic arch. the incidence of this type of supra aortic malformations is observed in $10 \%$ to $20 \%$ of the population according to recent imaging studies [4] The association with subaortic stenosis is rare. 


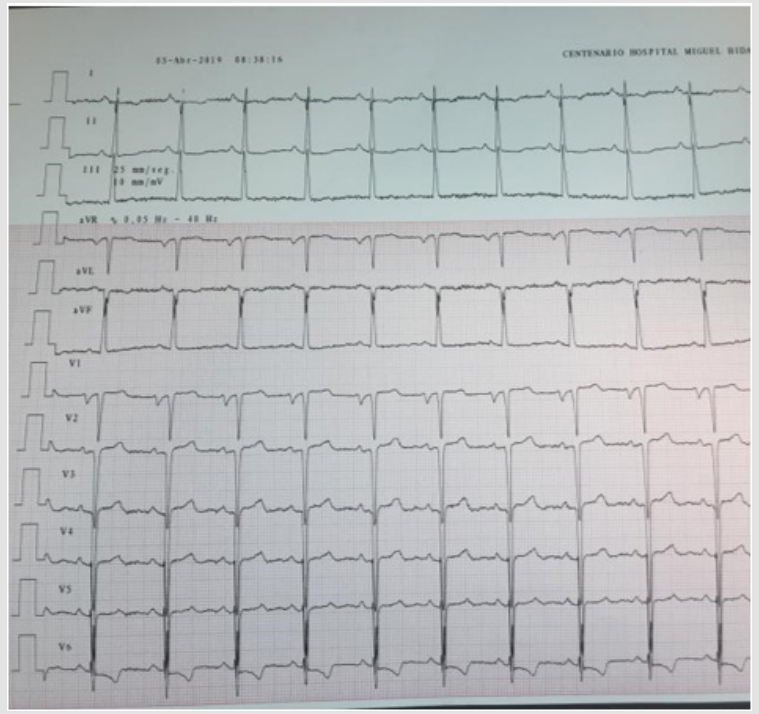

Figure 3: EKG with LVH and systolic overload.

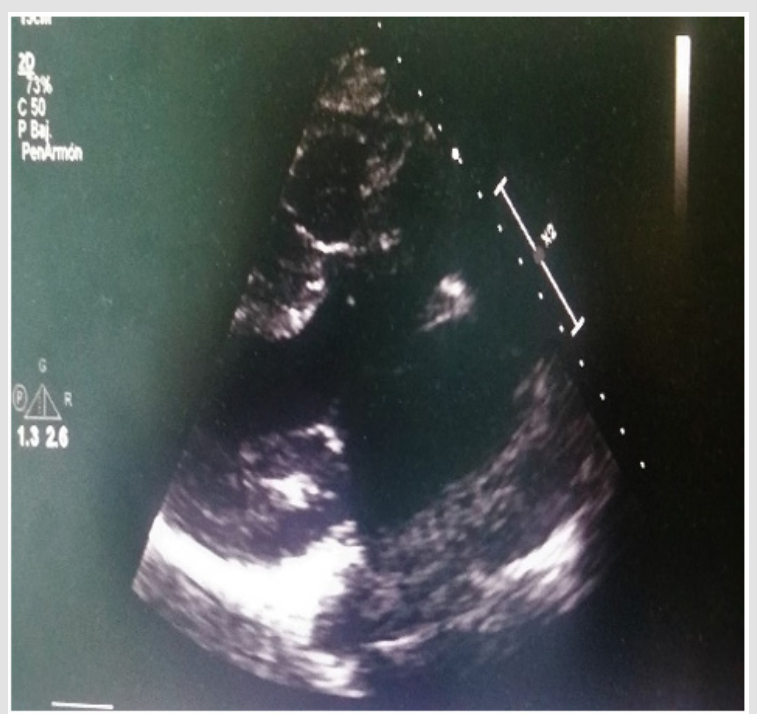

Figure 4: TEE subaortic fibromuscular impeller.

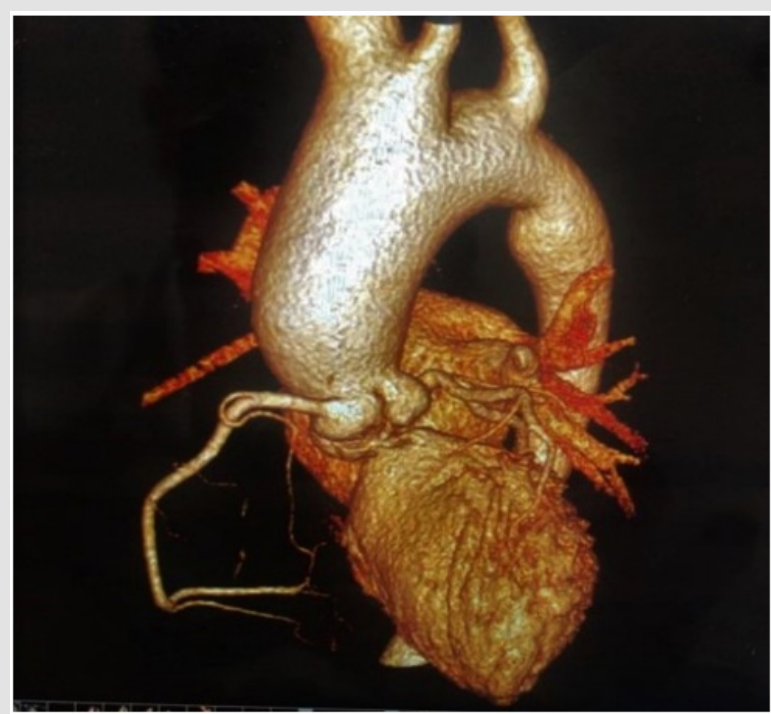

Figure 5: Aortic angiography. bovine aortic arch. 


\section{Discussion}

In $45-65 \%$ of patients, there is an association with other congenital cardiac defect, mainly ventricular septal defects, coarctation of the aorta, and bicuspid aortic valve. The LVOT obstruction progression is very variable; however, surgical correction is needed in most patients. SAS recurrence after surgical treatment is very frequent occurring in more than one-third of operated cases [5]. The clinical presentation of sub valvular aortic stenosis may be varied accompanied by other birth defects the first case presented subaortic stenosis is not accompanied by another congenital malformation the evolution is without complications so far, the second case is accompanied by persistence of the arterioscy duct that did not receive surgical treatment, is in protocol for definitive treatment and the third case the patient received surgical treatment of the closure of the arteries, however with severe aortic stenosis associated with a malformation of the supra aortic vessels (bovine aortic trunk) has been reported to be this association in very rare. The presence of throbbing headache may infer that it has a defect associated with these heart diseases, in patients with bovine aortic arch has been shown a significant association with the prevalence of thoracic disease $26 \%$ [6]. It is important to note that sub-aortic stenosis may have other associated congenital variants that may be relevant in clinical evolution and patient prognosis.

\section{Acknowledgement}

None.

\section{Conflict of Interest}

No conflict of interest.

\section{References}

1. Devabhaktuni SR, Chakfeh E, Malik AO, Pengson JA, Rana J, et al. (2018) Subvalvular aortic stenosis: a review of current literature. Clin Cardiol 419(1): 131-136.

2. Stout KK, Daniels CJ, Aboulhosn JA, Bozkurt B, Broberg CS, et al. (2019) 2018 AHA/ACC Guideline for the Management of Adults With Congenital Heart Disease: A Report of the American College of Cardiology/American Heart Association Task Force on Clinical Practice Guidelines. Circulation 139 (14): e698-e800.

3. Mulla S, Siddiqui WJ (2019) Subaortic Stenosis.

4. Malone CD, Urbania TH, Crook SES, Hope MD (2012) Bovine aortic arch: A novel association with thoracic aortic dilation, Clinical Radiology 67(1): 28-31.

5. Lopes R, Lourenço P, Gonçalves A, Cruz C, Maciel MJ (2011) The Natural History of Congenital Subaortic Stenosis. Congenital Heart Disease 6(5): 417-423

6. Hornick M, Moomiaie R, Mojibian H, Ziganshin B, Almuwaqqat Z, et al. (2012) 'Bovine' Aortic Arch -A Marker for Thoracic Aortic Disease. Cardiology 123(2): 116-124.
ISSN: 2574-1241

DOI: 10.26717/BJSTR.2019.21.003644

Delgado Leal L. Biomed J Sci \& Tech Res

(C) (P) This work is licensed under Creative

Submission Link: https://biomedres.us/submit-manuscript.php

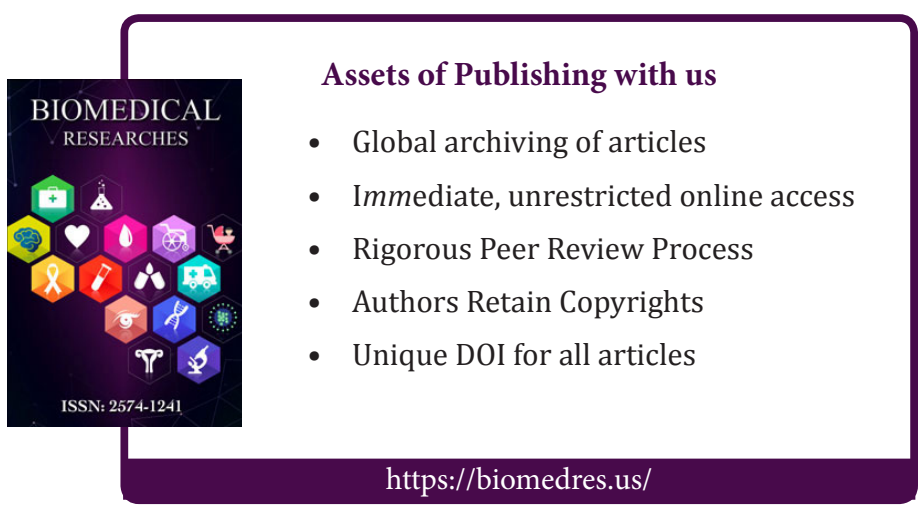

